Article

\title{
Association between Nutrients and Visceral Fat in Healthy Japanese Adults: A 2-Year Longitudinal Study Brief Title: Micronutrients Associated with Visceral Fat Accumulation
}

\author{
Naoki Ozato ${ }^{1,2, * \mathbb{D}}$, Shinichiro Saito ${ }^{3}$, Tohru Yamaguchi ${ }^{2}{ }^{\mathbb{D}}$, Mitsuhiro Katashima ${ }^{1,2}$, \\ Itoyo Tokuda ${ }^{4}$, Kaori Sawada ${ }^{4}$, Yoshihisa Katsuragi ${ }^{1,2}$, Seiya Imoto ${ }^{5}$, Kazushige Ihara 4 \\ and Shigeyuki Nakaji ${ }^{4}$ (D) \\ 1 Department of Active Life Promotion Sciences, Graduate School of Medicine, Hirosaki University, \\ Hirosaki 0368562, Japan; katashima.mitsuhiro@kao.com (M.K.); katsuragi.yoshihisa@kao.com (Y.K.) \\ 2 Health Care Food Research Laboratories, Kao Corporation, Tokyo 1318501, Japan; \\ yamaguchi.tohru@kao.com \\ 3 Biological Science Research Laboratories, Kao Corporation, Tokyo 1318501, Japan; saito.shinichiro@kao.com \\ 4 Department of Social Medicine, Graduate School of Medicine, Hirosaki University, Hirosaki 0368562, Japan; \\ i-tokuda@hirosaki-u.ac.jp (I.T.); iwane@hirosaki-u.ac.jp (K.S.); ihara@hirosaki-u.ac.jp (K.I.); \\ nakaji@hirosaki-u.ac.jp (S.N.) \\ 5 Health Intelligence Center, Institute of Medical Science, University of Tokyo, Tokyo 1088639, Japan; \\ imoto@ims.u-tokyo.ac.jp \\ * Correspondence: oozato.naoki@kao.com; Tel.: +81-(0)-17-239-5041
}

Received: 16 October 2019; Accepted: 5 November 2019; Published: 7 November 2019

\begin{abstract}
High visceral fat area (VFA) is a stronger predictor of cardiovascular disease and overall mortality than body mass index or waist circumference. VFA may be decreased by proper dietary habits. Although previous epidemiologic studies demonstrated an association between nutritional components or foodstuffs and VFA, only the associations of a few nutrients, such as dietary fiber and calcium, are reported. We performed a comprehensive 2-year longitudinal study in more than 624 healthy people and analyzed 33 micronutrients to investigate nutrients that contribute to changes in visceral fat. Our analyses revealed that "macronutrients" and "micronutrients" were "mutual confounders". Therefore, when evaluating the association between VFA and micronutrients, associations were adjusted by macronutrients. The ingestion of 7 nutrients: soluble dietary fiber, manganese, potassium, magnesium, vitamin $\mathrm{K}$, folic acid, and pantothenic acid, which are abundant components in vegetable diets, was significantly inversely correlated with a change in VFA. Additionally, a change in the ingestion of one nutrient, monounsaturated fat, was significantly positively correlated with a change in VFA. These associations were independent of body mass index and waist circumference. Thus, a predominantly vegetable diet may decrease VFA. In addition, adjusting the intake of macronutrients might help to clarify the association of micronutrients with VFA.
\end{abstract}

Keywords: micronutrients; visceral fat; obesity; BMI; macronutrients; vegetable

\section{Introduction}

Obesity results from an imbalance between energy consumption and expenditure. Therefore, increases in physical activity or proper dietary habits are recommended for maintaining an appropriate body weight [1]. Dietary habits are crucial in the development of overweight and obesity [2], and suboptimal diet can potentially be a major contributor to mortality in all countries worldwide [3]. Visceral fat accumulation, also known as visceral obesity, is a major predictor of cardiovascular 
disease [4] and all-cause mortality [5-7] independent of the body mass index (BMI) and general obesity. Clustering of metabolic risk factors, including hypertension, high blood glucose and triglyceride (TG) concentrations, and low serum high-density lipoprotein (HDL) cholesterol, is more strongly associated with the visceral fat area (VFA) than high subcutaneous fat, waist circumference (WC), or BMI $[8,9]$. Thus, evaluation of diets to reduce VFA is important to protect against poor metabolic status.

Some epidemiologic studies, including cross-sectional studies [10-19] and longitudinal studies [20-24], have investigated the association between nutritional components or foodstuff and VFA. These analyses indicated that only dietary fiber [17,21,23], carotenoid [15] and calcium [16,22] were negatively associated with VFA in terms of nutritional components. There are 2 analyses including over 1000 individuals; however, both studies targeted exclusively Westerners and patients [20,21]. We reported the association of visceral fat and nutrition in healthy Asians who eat mainly rice and tend to accumulate more visceral fat compared with Westerners [25] in a cross-sectional study [12,19].

In the present study, we performed a 2-year longitudinal study in 624 relatively healthy Japanese subjects, and comprehensively investigated the nutrients associated with the accumulation of visceral fat by assessing the relationship of 33 micronutrients and VFA.

\section{Materials and Methods}

\subsection{Study Subjects}

The Iwaki Health Promotion Project was launched in 2005 as an annual health check-up for local residents, aiming to prolong a healthy lifespan. Participants were men and women of at least 20 years of age living in the Iwaki region of Hirosaki City, Aomori Prefecture, Japan [26-29]. Inclusion criterion was individuals who have health condition ability to participate in this health check-up for more than $5 \mathrm{~h}$. VFA was first introduced as a health check-up parameter in 2015, and the present analyses were performed using data obtained from the health check-ups held from May 2015 to May 2017. In 2015, 1118 individuals participated in the health check-up (Figure 1). Of these, 36 participants did not complete the clinical assessments, dietary data, and/or VFA, and were excluded from the analyses. In addition, we excluded 458 participants with missing dietary and/or VFA data from 2016 and/or 2017. Thus, 624 individuals (260 men and 364 women; mean age \pm standard deviation, $54.0 \pm 14.2$ years for men and $55.1 \pm 13.6$ years for women) were enrolled into the analyses.

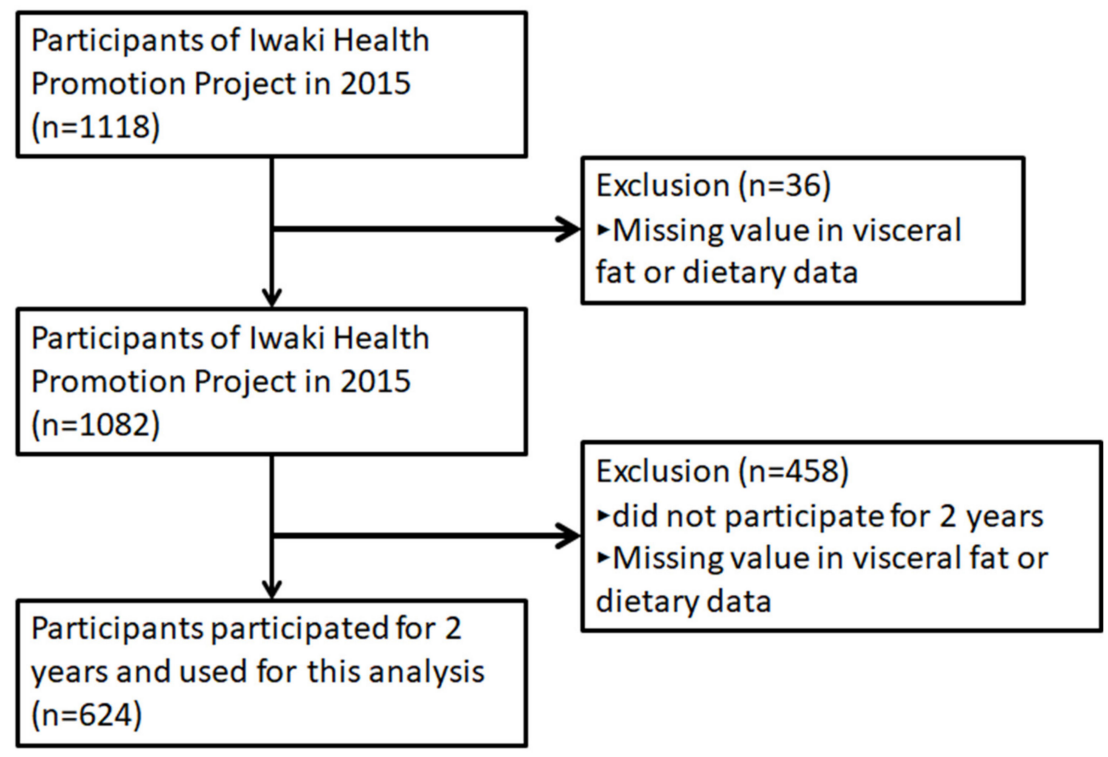

Figure 1. Study flow of the participants. A total of 624 participants completed measurements at the 3 time-points for 2 years (baseline in 2015, 2016, and 2017) among 1118 adults who participated in Iwaki Health Promotion Projects in 2015 (baseline) and were enrolled for the analysis. 


\subsection{Dietary Exposure}

A validated, self-administered, brief diet history questionnaire (BDHQ) was used to assess the dietary intake of the participants during the preceding month [30-32]. The BDHQ is a structured questionnaire that contains questions about the intake of approximately 58 foods and beverages, and allows for estimation of the total energy intake, the intake of 3 macronutrients (protein, fat, and carbohydrate), and the intake of 33 micronutrients. Two previous studies demonstrated that BDHQ-derived intake estimates are consistent with intake measured using semi-weighted 16-day dietary records [32,33]. The BDHQ has been used in cross-sectional [34] or cohort [35] studies. In the present study, we measured the dietary habits using the BDHQ at three time-points in a 2-year time period (May 2015 as baseline, May 2016, and May 2017).

\subsection{Measurements of Other Items}

All participants attended a health check-up early in the morning after fasting for at least $9 \mathrm{~h}$ at baseline and each subsequent year. VFA was measured using a visceral fat meter, the EW-FA90 (Panasonic Corporation, Osaka, Japan), which is an authorized medical device in Japan (No. 22500BZX00522000). This device produces results that highly correlate with those obtained using computed tomography (CT) [36], the gold standard for VFA measurement. As metabolic risk factors, the following clinical characteristics were measured: BMI (calculated from height and weight), WC, VFA, serum glucose, glycated hemoglobin (HbA1c), systolic blood pressure (SBP), diastolic blood pressure (DBP), serum TG, HDL-cholesterol, and low-density lipoprotein (LDL)-cholesterol. Blood samples were collected from peripheral veins. All laboratory tests were outsourced to LSI Medience Co. (Tokyo, Japan) according to the instructions of the vendors. Smoking habit (cigarettes/d), sleep time (h/d), and amount of exercise (mets/d) were determined from questionnaires. Amount of exercise was calculated using Mets conversion table by following questionnaire items; type of exercise, number of times per week, and hours per time [37].

\subsection{Statistical Analysis}

Characteristics of the study participants are reported as means \pm standard deviation (SD). At baseline, the relationship between VFA and each value was tested for linearity using a multivariate model, including age, smoking habit, amount of exercise, BMI, and WC, which are well-known confounding factors of VFA and dietary habits. The amount of change in each nutrient and VFA was calculated from the slope of the three time-points obtained for each parameter. We hypothesized that a clearer association between micronutrients and VFA could be obtained by adjusting for related confounders. The associations between change in VFA and change in each macronutrient were assessed by a multiple regression analysis with change in VFA as the objective variable, and change in the macronutrient and covariates, such as age, sex, VFA, and amount of each macronutrient ingested at baseline; and change in the amount of exercise, BMI, WC, smoking habit, and energy ingested as explanatory variables. The associations between change in VFA and change in each micronutrient were assessed by multiple regression analysis with change in VFA as the objective variable, and change in each micronutrient and covariates, such as age, sex, VFA, and intake amount of each macronutrient (protein, fat, and carbohydrate) at baseline; and change in the amount of each macronutrient (protein, fat, and carbohydrate) ingested, amount of exercise, BMI, WC, smoking habit, and amount of energy ingested as explanatory variables. The associations between change in BMI and change in each micronutrient were assessed using multiple regression analysis with change in BMI as the objective variable, and change in each micronutrient and covariates, such as age, sex, BMI, and amount of each macronutrient (protein, fat, and carbohydrate) ingested at baseline; and change in the amount of each macronutrient (protein, fat, and carbohydrate) ingested, amount of exercise, VFA, WC, smoking habits, and amount of energy ingested as explanatory variables. 
To investigate the association between the change in each macronutrient and the change in each micronutrient, the correlation coefficient and its $\mathrm{P}$ value were estimated by the Spearman correlation test. Statistical tests were two-sided, and values of $p<0.05$ were considered statistically significant. All analyses were performed using the R software version 3.3.4.

\subsection{Ethics Statement}

This study was performed in accordance with the ethical standards of the Declaration of Helsinki and approved by the ethics committee at Hirosaki University Medical Ethics Committee (2014-377, 2016-028, and 2017-026). Written informed consent was obtained from all participants prior to the study. This study was registered at the University Hospital Medical Information Network (UMIN-CTR, https://www.umin.ac.jp) prior to the analyses (UMIN ID: UMIN000030351).

\section{Results}

\subsection{Baseline Characteristics Based on the Cut-Off Point for Visceral Obesity}

Baseline characteristics of the study sample $(\mathrm{N}=624,58 \%$ female $)$ are summarized in Table 1. The rate of overweight (defined as BMI $\geq 25$ ) was $29.2 \%$ in men and $16.2 \%$ in women. The rates were comparable to those of the 2010 national survey reported by the Japanese government (overweight and obesity rate in subjects 30-69 years of age: $33.5 \%$ in men and 20.5\% in women). Mean VFA was $106.8 \pm 42.7 \mathrm{~cm}^{2}$ in men and $66.4 \pm 32.0 \mathrm{~cm}^{2}$ in women, and was higher in men and lower in women than the value defined as visceral obesity $\left(\geq 100 \mathrm{~cm}^{2}\right)$ by the Japan Society for the Study of Obesity [38]. The individuals were divided into two groups based on the cut-off point for VFA, the VFA+ group (VFA $\geq 100,144$ men and 45 women) or the VFA-group (VFA < 100, 116 men and 319 women; Table 1). As for metabolic risk factors, glucose, $\mathrm{HbA1c}$, SBP, DBP, TG, and LDL cholesterol values were significantly higher in the VFA+ group, while the HDL cholesterol values were significantly lower regardless of $\operatorname{sex}(p<0.001,<0.001,<0.001,0.021,<0.001,<0.001$, and 0.001 for men and $<0.001,<0.001,<0.001$, $<0.001,<0.001,<0.001$, and 0.001 for women, respectively). As for dietary habits, intake of energy or macronutrients was not significantly associated with VFA. Furthermore, in men, only retinol intake was significantly inversely associated with VFA $(p=0.048)$, and in women, only sodium intake was significantly positively associated with VFA $(p=0.033)$, but no other micronutrient examined was significantly associated with VFA, regardless of sex, in the baseline study.

Table 1. Baseline participants' characteristics based on the cut-off point of visceral obesity.

\begin{tabular}{|c|c|c|c|c|c|c|}
\hline \multirow[b]{2}{*}{ Characteristics } & \multicolumn{3}{|c|}{ MEN } & \multicolumn{3}{|c|}{ WOMEN } \\
\hline & VFA $<100$ & $100 \leq$ VFA & $p^{a}$ & VFA $<100$ & $100 \leq \mathrm{VFA}$ & $p^{a}$ \\
\hline Number of participants & 116 & 144 & & 319 & 45 & \\
\hline Age $(\mathrm{yr})$ & $52.1 \pm 14.5$ & $55.5 \pm 13.8$ & & $54.5 \pm 13.7$ & $59.5 \pm 11.6$ & - \\
\hline \multicolumn{7}{|l|}{ Metabolic risk factors: } \\
\hline Body mass index $(\mathrm{kg} / \mathrm{m} 2)$ & $21.7 \pm 2.0$ & $25.2 \pm 2.7$ & - & $21.4 \pm 2.7$ & $27.1 \pm 3.6$ & - \\
\hline Waist circumference $(\mathrm{cm})$ & $76.9 \pm 5.1$ & $87.7 \pm 6.6$ & - & $71.8 \pm 7.3$ & $87.1 \pm 7.7$ & - \\
\hline Visceral fat mass $\left(\mathrm{cm}^{2}\right)$ & $6.9 \pm 17.5$ & $137.1 \pm 28.8$ & - & $57.9 \pm 22.8$ & $125.8 \pm 24.6$ & - \\
\hline Glucose $(\mathrm{mmol} / \mathrm{l})$ & $4.5 \pm 0.6$ & $5.0 \pm 1.1$ & $<0.001^{* * *}$ & $4.4 \pm 0.7$ & $4.8 \pm 0.7$ & $<0.001^{* * *}$ \\
\hline $\operatorname{HbA} 1 \mathrm{c}(\%)$ & $5.6 \pm 0.4$ & $5.9 \pm 0.8$ & $<0.001^{* * *}$ & $5.7 \pm 0.5$ & $6.0 \pm 0.7$ & $<0.001^{* * *}$ \\
\hline $\mathrm{SBP}(\mathrm{mmHg})$ & $122.5 \pm 18.1$ & $128.2 \pm 16.6$ & $<0.001^{* * * *}$ & $117.3 \pm 16.4$ & $130.2 \pm 16.9$ & $<0.001^{* * *}$ \\
\hline $\mathrm{DBP}(\mathrm{mmHg})$ & $77.4 \pm 12.6$ & $79.2 \pm 10.2$ & $0.021 *$ & $71.8 \pm 10.3$ & $76.3 \pm 10.4$ & $<0.001^{* * *}$ \\
\hline $\mathrm{TG}(\mathrm{mmol} / \mathrm{l})$ & $1.1 \pm 0.7$ & $1.7 \pm 1.4$ & $<0.001^{* * * *}$ & $0.9 \pm 0.4$ & $1.2 \pm 0.5$ & $<0.001^{* * *}$ \\
\hline HDL-cholesterol (mmol/l) & $1.7 \pm 0.4$ & $1.5 \pm 0.5$ & $<0.001^{* * *}$ & $1.9 \pm 0.4$ & $1.6 \pm 0.3$ & $<0.001^{* * *}$ \\
\hline LDL-cholesterol (mmol/l) & $2.9 \pm 0.7$ & $3.1 \pm 0.7$ & $0.001^{* *}$ & $3.0 \pm 0.8$ & $3.2 \pm 0.7$ & $0.001 * *$ \\
\hline \multicolumn{7}{|l|}{ Lifestyle habits: } \\
\hline Smoking habits (stick/d) & $12.8 \pm 17.8$ & $11.5 \pm 11.9$ & - & $2.3 \pm 5.3$ & $2.1 \pm 4.9$ & - \\
\hline Sleep time $(\mathrm{h} / \mathrm{d})$ & $7.0 \pm 1.2$ & $7.3 \pm 1.2$ & - & $6.7 \pm 1.0$ & $7.1 \pm 0.9$ & - \\
\hline Amount of exercise (Mets/d) & $6.9 \pm 17.5$ & $8.4 \pm 18.4$ & - & $4.3 \pm 10.7$ & $6.0 \pm 10.8$ & - \\
\hline \multicolumn{7}{|l|}{ Dietary habits: } \\
\hline Energy (kcal/d) & $\begin{array}{c}2189.1 \pm \\
667.7\end{array}$ & $\begin{array}{c}2107.3 \pm \\
543.7\end{array}$ & 0.331 & $1655 \pm 439$ & $1752 \pm 503$ & 0.517 \\
\hline Macronutrient: & & & & & & \\
\hline
\end{tabular}


Table 1. Cont.

\begin{tabular}{|c|c|c|c|c|c|c|}
\hline \multirow[b]{2}{*}{ Characteristics } & \multicolumn{3}{|c|}{ MEN } & \multicolumn{3}{|c|}{ WOMEN } \\
\hline & VFA $<100$ & $100 \leq$ VFA & $p^{\mathrm{a}}$ & VFA $<100$ & $100 \leq \mathrm{VFA}$ & $p^{\mathrm{a}}$ \\
\hline Protein $(\mathrm{g} / \mathrm{d})$ & $76.1 \pm 29.6$ & $74.5 \pm 24.5$ & 0.422 & $64.0 \pm 22.1$ & $67.8 \pm 25.8$ & 0.489 \\
\hline Carbohydrate (g/d) & $303.1 \pm 101.9$ & $281.8 \pm 82.4$ & 0.115 & $225.8 \pm 63.0$ & $242.8 \pm 64.9$ & 0.130 \\
\hline \multicolumn{7}{|l|}{ Micronutrient: } \\
\hline Ash (g/d) & $19.6 \pm 6.7$ & $19.6 \pm 5.7$ & 0.647 & $16.5 \pm 5.0$ & $17.8 \pm 6.0$ & 0.184 \\
\hline Calcium (mg/d) & $483 \pm 209$ & $519 \pm 236$ & 0.539 & $480 \pm 196$ & $490 \pm 210$ & 0.666 \\
\hline Magnesium (mg/d) & $269 \pm 99$ & $267 \pm 89$ & 0.692 & $232 \pm 78$ & $237 \pm 85$ & 0.633 \\
\hline Phosphorus (mg/d) & $1109 \pm 420$ & $1105 \pm 373$ & 0.614 & $957 \pm 331$ & $1005 \pm 387$ & 0.481 \\
\hline Iron $(\mathrm{mg} / \mathrm{d})$ & $8.0 \pm 3.6$ & $7.8 \pm 2.8$ & 0.495 & $7.1 \pm 2.6$ & $7.2 \pm 2.9$ & 0.839 \\
\hline Zinc (mg/d) & $9.0 \pm 3.2$ & $8.8 \pm 2.5$ & 0.601 & $7.5 \pm 2.3$ & $7.9 \pm 2.6$ & 0.522 \\
\hline Copper $(\mathrm{mg} / \mathrm{d})$ & $1.3 \pm 0.5$ & $1.3 \pm 0.4$ & 0.503 & $1.1 \pm 0.3$ & $1.1 \pm 0.3$ & 0.410 \\
\hline Vitamin D $(\mu \mathrm{g} / \mathrm{d})$ & $15.1 \pm 10.7$ & $14.3 \pm 9.8$ & 0.155 & $13.4 \pm 9.2$ & $14.3 \pm 11.9$ & 0.310 \\
\hline$\alpha$-tocopherol $(\mathrm{mg} / \mathrm{d})$ & $7.1 \pm 3.3$ & $6.9 \pm 2.5$ & 0.676 & $6.5 \pm 2.5$ & $6.5 \pm 2.7$ & 0.544 \\
\hline Vitamin $K(\mu \mathrm{g} / \mathrm{d})$ & $320.3 \pm 172.0$ & $322.0 \pm 167.1$ & 0.633 & $303 \pm 161$ & $282 \pm 143$ & 0.484 \\
\hline Thiamin (mg/d) & $0.7 \pm 0.3$ & $0.7 \pm 0.3$ & 0.798 & $0.7 \pm 0.2$ & $0.7 \pm 0.3$ & 0.797 \\
\hline Riboflavin (mg/d) & $1.3 \pm 0.6$ & $1.3 \pm 0.5$ & 0.694 & $1.2 \pm 0.4$ & $1.2 \pm 0.4$ & 0.839 \\
\hline $\operatorname{Niacin}(\mathrm{mg} / \mathrm{d})$ & $19.2 \pm 8.2$ & $18.2 \pm 7.7$ & 0.219 & $15.6 \pm 6.2$ & $15.6 \pm 6.8$ & 0.812 \\
\hline Vitamin $B_{6}(\mathrm{mg} / \mathrm{d})$ & $1.3 \pm 0.6$ & $1.3 \pm 0.5$ & 0.582 & $1.1 \pm 0.4$ & $1.1 \pm 0.5$ & 0.805 \\
\hline Vitamin $B_{12}(\mu \mathrm{g} / \mathrm{d})$ & $11.5 \pm 8.3$ & $10.4 \pm 6.1$ & 0.091 & $9.1 \pm 5.5$ & $9.7 \pm 6.4$ & 0.324 \\
\hline Folate $(\mu \mathrm{g} / \mathrm{d})$ & $317.1 \pm 158.7$ & $306.3 \pm 130.2$ & 0.616 & $282 \pm 126$ & $274 \pm 117$ & 0.659 \\
\hline Pantothenic acid (mg/d) & $6.8 \pm 2.6$ & $6.8 \pm 2.2$ & 0.848 & $5.9 \pm 2.0$ & $6.1 \pm 2.1$ & 0.658 \\
\hline Vitamin C (mg/d) & $83.6 \pm 52.2$ & $86.0 \pm 53.2$ & 0.584 & $85.9 \pm 47.6$ & $87.9 \pm 49.0$ & 0.804 \\
\hline Insoluble dietary fiber $(\mathrm{g} / \mathrm{d})$ & $8.5 \pm 3.7$ & $8.4 \pm 3.2$ & 0.990 & $7.8 \pm 2.9$ & $7.9 \pm 2.9$ & 0.682 \\
\hline Alcohol $(\mathrm{g} / \mathrm{d})$ & $19.5 \pm 21.2$ & $22.1 \pm 22.6$ & 0.236 & $4.2 \pm 10.6$ & $5.0 \pm 11.6$ & 0.517 \\
\hline Daidzein (mg/d) & $15.8 \pm 10.3$ & $16.7 \pm 10.0$ & 0.714 & $15.3 \pm 9.2$ & $15.8 \pm 8.4$ & 0.700 \\
\hline Genistein (mg/d) & $26.7 \pm 17.3$ & $28.3 \pm 17.0$ & 0.720 & $25.9 \pm 15.5$ & $26.8 \pm 14.2$ & 0.684 \\
\hline
\end{tabular}

$p<0.05, p<0.01$ and $p<0.001$ are indicated by "**, "***, and “***”, respectively. Data are mean \pm SD. ${ }^{a} p$ value was adjusted by age, smoking habit, amount of exercise, BMI, and WC. Abbreviations: VFA, visceral fat area. BMI, body mass index. WC, waist circumference. SBP, systolic blood pressure. DBP, diastolic blood pressure, TG, triglyceride. HDL-cholesterol, high-density lipoprotein- cholesterol. LDL- cholesterol, low-density lipoprotein- cholesterol.

\subsection{Association Between Changes in VFA and Macronutrient Intake Over 2 Years}

The association between changes in visceral fat and intake of macronutrients over 2 years is shown in Table 2 adjusted by Models 1 and 2. In Model 1, the adjustment was performed for the following factors: age, sex, VFA, and amount of each macronutrient (protein, fat, and carbohydrate) ingested at baseline, and amount of change in exercise, BMI, WC, and smoking habit. The change in VFA and in intake of energy, proteins, fats, or carbohydrates was not significantly associated $(\beta=0.002$, $p=0.239, \beta=0.043, p=0.193, \beta=0.057, p=0.186$, and $\beta=0.005, p=0.632$, respectively). In Model 2, change in energy intake was added to Model 1 as the confounding factor for the adjustment. Similar to the above-mentioned results, no significant relationship was detected between change in VFA and change in the intake amount of protein, fat, or carbohydrate $(\beta=0.033, p=0.548, \beta=0.042, p=0.527$, and $\beta=-0.035, p=0.108$, respectively). 
Table 2. Association between VFA and macronutrient change over 2 years.

\begin{tabular}{|c|c|c|c|c|c|c|c|c|c|}
\hline \multirow[b]{2}{*}{ Characteristics } & \multicolumn{3}{|c|}{ Change in VFA } & \multicolumn{3}{|c|}{ Adjusted by Model $1^{\text {a }}$} & \multicolumn{3}{|c|}{ Adjusted by Model $2^{b}$} \\
\hline & Q1 & Q2 & Q3 & \multicolumn{2}{|c|}{$\beta$ (s.e.) } & $p$ & \multicolumn{2}{|c|}{$\beta$ (s.e.) } & $p$ \\
\hline Number of participants & 203(79/124) & $213(67 / 146)$ & 208(114/94) & & & & & & \\
\hline Baseline age $(\mathrm{y})$ & $55.0 \pm 13.6$ & $54.7 \pm 14.6$ & $54.2 \pm 13.3$ & & & & & & \\
\hline Visceral fat mass $\left(\Delta \mathrm{cm}^{2}\right)$ & $-9.1 \pm 5.2$ & $0.6 \pm 2.2$ & $10.9 \pm 6.7$ & & & & & & \\
\hline Energy $(\Delta \mathrm{kcal} / \mathrm{d})$ & $-1.5 \pm 226.4$ & $15.0 \pm 199.7$ & $21.2 \pm 224.3$ & 0.002 & $(0.002)$ & 0.239 & - & - & - \\
\hline Macronutrient: & & & & & & & & & \\
\hline $\operatorname{Protein}(\Delta \mathrm{g} / \mathrm{d})$ & $0.72 \pm 10.80$ & $2.29 \pm 10.26$ & $0.99 \pm 11.17$ & 0.043 & $(0.033)$ & 0.193 & 0.033 & $(0.055)$ & 0.548 \\
\hline Fat $(\Delta \mathrm{g} / \mathrm{d})$ & $0.62 \pm 8.78$ & $0.97 \pm 7.78$ & $0.82 \pm 8.56$ & 0.057 & $(0.043)$ & 0.186 & 0.042 & $(0.066)$ & 0.527 \\
\hline Carbohydrate $(\Delta \mathrm{g} / \mathrm{d})$ & $-2.83 \pm 31.90$ & $-1.10 \pm 29.38$ & $0.99 \pm 34.22$ & 0.005 & $(0.011)$ & 0.632 & -0.035 & $(0.022)$ & 0.108 \\
\hline
\end{tabular}

\subsection{Association Between Macronutrients and Micronutrients}

The associations between changes in macronutrients and micronutrients are shown in Table 3. Each macronutrient (protein, fat, and carbohydrate) significantly correlated with almost all of the micronutrients except for alcohol, suggesting that "macronutrients" and "micronutrients" could be mutual confounding factors. Therefore, we consider that the direct or indirect correlation with VFA cannot be estimated without adjusting for the other factor. That is, when examining the association of VFA with micronutrients or macronutrients, they must be adjusted for each other.

Table 3. Association between Macronutrient and Micronutrient change over 2 years.

\begin{tabular}{|c|c|c|c|}
\hline & \multicolumn{3}{|c|}{ Macronutrient } \\
\hline & Protein & Fat & Carbohydrate \\
\hline Characteristics & \multicolumn{3}{|c|}{ Correlation Coefficient $(r)^{a}$} \\
\hline Micronutrient: & & & \\
\hline $\operatorname{Ash}(\Delta \mathrm{g} / \mathrm{d})$ & $0.88^{* * *}$ & $0.71^{* * *}$ & $0.54^{* * *}$ \\
\hline Sodium $(\Delta \mathrm{mg} / \mathrm{d})$ & $0.78^{* * *}$ & $0.61^{* * *}$ & $0.50 * * *$ \\
\hline Potassium $(\Delta \mathrm{mg} / \mathrm{d})$ & $0.79^{* * *}$ & $0.70 * * *$ & $0.49^{* * *}$ \\
\hline Calcium $(\Delta \mathrm{mg} / \mathrm{d})$ & $0.69^{* * *}$ & $0.60^{* * *}$ & $0.39^{* * *}$ \\
\hline Magnesium $(\Delta \mathrm{mg} / \mathrm{d})$ & $0.85^{* * *}$ & $0.70^{* * *}$ & $0.56^{* * *}$ \\
\hline Phosphorus ( $\Delta \mathrm{mg} / \mathrm{d})$ & $0.96^{* * *}$ & $0.81^{* * *}$ & $0.53^{* * *}$ \\
\hline $\operatorname{Iron}(\Delta \mathrm{mg} / \mathrm{d})$ & $0.84^{* * *}$ & $0.72 * * *$ & $0.48^{* * *}$ \\
\hline $\operatorname{Zinc}(\Delta \mathrm{mg} / \mathrm{d})$ & $0.92^{* * *}$ & $0.79 * * *$ & $0.63^{* * *}$ \\
\hline Copper $(\Delta \mathrm{mg} / \mathrm{d})$ & $0.80^{* * *}$ & $0.61^{* * *}$ & $0.72 * * *$ \\
\hline Manganese $(\Delta \mathrm{mg} / \mathrm{d})$ & $0.43^{* * *}$ & $0.32 * * *$ & $0.60 * * *$ \\
\hline Vitamin $\mathrm{A}$ (retinol equivalent) $(\Delta \mu \mathrm{g} / \mathrm{d})$ & $0.45^{* * *}$ & $0.45^{* * *}$ & $0.21^{* * *}$ \\
\hline Retinol $(\Delta \mu \mathrm{g} / \mathrm{d})$ & $0.47^{* * *}$ & $0.46^{* * *}$ & $0.19^{* * *}$ \\
\hline$\beta$-carotene equivalente $(\Delta \mu \mathrm{g} / \mathrm{d})$ & $0.37^{* * *}$ & $0.32^{* * *}$ & $0.21^{* * *}$ \\
\hline $\operatorname{Vitamin} \mathrm{D}(\Delta \mu \mathrm{g} / \mathrm{d})$ & $0.70^{* * *}$ & $0.51^{* * *}$ & $0.21^{* * *}$ \\
\hline$\alpha$-tocopherol $(\Delta \mathrm{mg} / \mathrm{d}))$ & $0.79^{* * *}$ & $0.84^{* * *}$ & $0.41^{* * *}$ \\
\hline Vitamin $\mathrm{K}(\Delta \mu \mathrm{g} / \mathrm{d})$ & $0.50^{* * *}$ & $0.47^{* * *}$ & $0.25 * * *$ \\
\hline Thiamin $(\Delta \mathrm{mg} / \mathrm{d})$ & $0.85^{* * *}$ & $0.80^{* * *}$ & $0.52 * * *$ \\
\hline Riboflavin $(\Delta \mathrm{mg} / \mathrm{d})$ & $0.82^{* * *}$ & $0.77^{* * *}$ & $0.41^{* * *}$ \\
\hline $\operatorname{Niacin}(\Delta \mathrm{mg} / \mathrm{d})$ & $0.85^{* * *}$ & $0.73^{* * *}$ & $0.36^{* * *}$ \\
\hline Vitamin B6 $(\Delta \mathrm{mg} / \mathrm{d})$ & $0.87^{* * *}$ & $0.76^{* * *}$ & $0.46^{* * *}$ \\
\hline $\operatorname{Vitamin} \mathrm{B} 12(\Delta \mathrm{mg} / \mathrm{d})$ & $0.71^{* * *}$ & $0.53^{* * *}$ & $0.19^{* * *}$ \\
\hline Folate $(\Delta \mu \mathrm{g} / \mathrm{d})$ & $0.58^{* * *}$ & $0.52^{* * *}$ & $0.37^{* * *}$ \\
\hline Pantothenic acid $(\Delta \mathrm{mg} / \mathrm{d})$ & $0.90^{* * *}$ & $0.80^{* * *}$ & $0.58^{* * *}$ \\
\hline $\operatorname{Vitamin} C(\Delta \mathrm{mg} / \mathrm{d})$ & $0.44^{* * *}$ & $0.41^{* * *}$ & $0.35^{* * *}$ \\
\hline Saturated fat $(\Delta \mathrm{g} / \mathrm{d})$ & $0.74^{* * *}$ & $0.91^{* * *}$ & $0.42^{* * *}$ \\
\hline
\end{tabular}


Table 3. Cont.

\begin{tabular}{cccc}
\hline & \multicolumn{3}{c}{ Macronutrient } \\
\hline Characteristics & Protein & Fat & Carbohydrate \\
\hline Monounsaturated fat $(\Delta \mathrm{g} / \mathrm{d})$ & \multicolumn{2}{c}{ Correlation Coefficient $(\mathrm{r})^{\mathbf{a}}$} \\
\hline Polyunsaturated fat $(\Delta \mathrm{g} / \mathrm{d})$ & $0.79^{* * *}$ & $0.98^{* * *}$ & $0.38^{* * *}$ \\
$\mathrm{n}-3$ polyunsaturated fat $(\Delta \mathrm{g} / \mathrm{d})$ & $0.77^{* * *}$ & $0.90^{* * *}$ & $0.38^{* * *}$ \\
$\mathrm{n}-6$ polyunsaturated fat $(\Delta \mathrm{g} / \mathrm{d})$ & $0.78^{* * *}$ & $0.81^{* * *}$ & $0.30^{* * *}$ \\
Cholesterol $(\Delta \mathrm{mg} / \mathrm{d})$ & $0.72^{* * *}$ & $0.87^{* * *}$ & $0.39^{* * *}$ \\
Total dietary fiber $(\Delta \mathrm{g} / \mathrm{d})$ & $0.80^{* * *}$ & $0.72^{* * *}$ & $0.32^{* * *}$ \\
Soluble dietary fiber $(\Delta \mathrm{g} / \mathrm{d})$ & $0.59^{* * *}$ & $0.49^{* * *}$ & $0.56^{* * *}$ \\
Insoluble dietary fiber $(\Delta \mathrm{g} / \mathrm{d})$ & $0.54^{* * *}$ & $0.46^{* * *}$ & $0.47^{* * *}$ \\
Alcohol $(\Delta \mathrm{g} / \mathrm{d})$ & $0.59^{* * *}$ & $0.49^{* * *}$ & $0.59^{* * *}$ \\
Daidzein $(\Delta \mathrm{mg} / \mathrm{d})$ & $0.02^{* * *}$ & $0.07^{* * *}$ & $0.06^{* * *}$ \\
Genistein $(\Delta \mathrm{mg} / \mathrm{d})$ & $0.42^{* * *}$ & $0.35^{* * *}$ & $0.21^{* * *}$ \\
\hline
\end{tabular}

$p<0.001$ are indicated by “***”, respectively. Data are shown as mean $\pm \mathrm{SD} .{ }^{\text {a Correlation coefficient and } p}$ value were analyzed by Spearman's rank partial correlation analysis.

\subsection{Macronutrients Associated with Change in VFA or BMI}

The associations between changes in macronutrients and VFA or BMI are shown in Table 4. For VFA, in Model 1, the following factors were used for the adjustment: age, sex, VFA, and amount of each macronutrient (protein, fat, and carbohydrate) ingested at baseline, and the change in intake of energy, amount of exercise, BMI, WC, and smoking habit. Of the 33 micronutrients, changes in two nutrients, soluble dietary fiber and manganese, were significantly negatively associated with change in VFA ( $\beta=-2.31, p=0.014$ and $\beta=-2.43, p=0.018$ ) over 2 years. In Model 2 , the following factors were used for the adjustment: age, sex, VFA, and amount of each macronutrient (protein, fat, and carbohydrate) ingested at baseline, and the change in the amount of protein, fat, and carbohydrate ingested; amount of exercise; BMI; WC; and smoking habit. Of the 33 micronutrients, change in 7 micronutrients, soluble dietary fiber, manganese, potassium, magnesium, vitamin $\mathrm{K}$, folic acid, and pantothenic acid, were significantly negatively associated with change in VFA $(\beta=-2.59, p=0.007, \beta=-2.19, p=$ $0.042, \beta=-0.004, p=0.039, \beta=-0.06, p=0.018, \beta=-0.011, p=0.050, \beta=-0.018, p=0.045$ and $\beta=-3.43, p=0.001$ ), and change in 1 micronutrient, monounsaturated fat, was significantly positively associated with change in VFA $(\beta=1.34, p=0.050)$. However, vitamin $\mathrm{K}$ and monounsaturated fat had borderline p-values $(p=0.05)$. For BMI, in Model 3, the following factors were used for the adjustment: age, sex, BMI, and amount of each macronutrient (protein, fat, and carbohydrate) ingested at baseline, and change in the energy ingested, amount of exercise, VFA, WC, and smoking habit. Of the 33 micronutrients, none were significantly associated with a change in BMI. In Model 4 , however, the following factors were used for the adjustment: age, sex, BMI, and amount of each macronutrient (protein, fat, and carbohydrate) ingested at baseline, and change in the amount of protein, fat, and carbohydrate ingested; amount of exercise; VFA; WC; smoking habit; and amount of energy ingested. Of the 33 micronutrients, changes in 4 nutrients: alpha-tocopherol, vitamin B6, vitamin $\mathrm{B} 12$, and $\mathrm{n}-3$ polyunsaturated fat, were significantly negatively associated with change in VFA $(\beta=-0.061, p=0.023, \beta=-0.474, p=0.015, \beta=-0.020, p=0.015$, and $\beta=-0.115, p=0.031$ ). Taken together, 7 micronutrients: soluble dietary fiber, manganese, potassium, magnesium, vitamin $\mathrm{K}$, folic acid, pantothenic acid, alpha-tocopherol, vitamin B6, vitamin B12, and $\mathrm{n}-3$ polyunsaturated fat, which are abundant nutrients in a vegetable diet, had a significant inverse correlation to VFA. Only monounsaturated fat had a significant positive association with VFA. 
Table 4. Association between VFA and micronutrient change over 2 years.

\begin{tabular}{|c|c|c|c|c|c|c|c|c|c|c|c|c|}
\hline \multirow{4}{*}{$\begin{array}{c}\text { Characteristics } \\
\text { Micronutrient: } \\
\text { Ash }(\Delta \mathrm{g} / \mathrm{d})\end{array}$} & \multicolumn{6}{|c|}{ Change in VFA } & \multicolumn{6}{|c|}{ Change in BMI } \\
\hline & \multicolumn{3}{|c|}{ Adjusted by Model 1} & \multicolumn{3}{|c|}{ Adjusted by Model 2} & \multicolumn{3}{|c|}{ Adjusted by Model 3} & \multicolumn{3}{|c|}{ Adjusted by Model 4} \\
\hline & \multicolumn{2}{|c|}{$\beta$ (s.e.) } & \multirow{2}{*}{$\begin{array}{c}p \\
0.904\end{array}$} & \multicolumn{2}{|c|}{$\beta$ (s.e.) } & \multirow{2}{*}{$\begin{array}{c}p \\
0.369\end{array}$} & \multicolumn{2}{|c|}{$\beta$ (s.e.) } & \multirow{2}{*}{$\begin{array}{c}\boldsymbol{p} \\
0.956\end{array}$} & \multicolumn{2}{|c|}{$\beta$ (s.e.) } & \multirow{2}{*}{$\begin{array}{c}p \\
0.727\end{array}$} \\
\hline & -0.03 & $(0.24)$ & & -0.32 & $(0.36)$ & & -0.001 & $(0.011)$ & & -0.005 & $(0.016)$ & \\
\hline Sodium $(\Delta \mathrm{mg} / \mathrm{d})$ & 0.00 & $(0.00)$ & 0.694 & 0.00 & $(0.00)$ & 0.949 & 0.000 & $(0.000)$ & 0.957 & 0.000 & $(0.000)$ & 0.965 \\
\hline $\operatorname{Potassium}(\Delta \mathrm{mg} / \mathrm{d})$ & -0.00 & $(0.00)$ & 0.250 & -0.01 & $(0.00)$ & 0.039 * & 0.000 & $(0.000)$ & 0.682 & 0.000 & $(0.000)$ & 0.455 \\
\hline Calcium $(\Delta \mathrm{mg} / \mathrm{d})$ & 0.00 & $(0.00)$ & 0.372 & -0.01 & $(0.01)$ & 0.134 & 0.000 & $(0.000)$ & 0.524 & 0.000 & $(0.000)$ & 0.605 \\
\hline Magnesium $(\Delta \mathrm{mg} / \mathrm{d})$ & -0.02 & $(0.02)$ & 0.209 & -0.06 & $(0.02)$ & 0.018 * & 0.000 & $(0.001)$ & 0.565 & -0.001 & $(0.001)$ & 0.272 \\
\hline Phosphorus $(\Delta \mathrm{mg} / \mathrm{d})$ & 0.00 & $(0.00)$ & 0.951 & -0.01 & $(0.01)$ & 0.111 & 0.000 & $(0.000)$ & 0.797 & 0.000 & $(0.000)$ & 0.724 \\
\hline $\operatorname{Iron}(\Delta \mathrm{mg} / \mathrm{d})$ & -0.34 & $(0.44)$ & 0.445 & -1.06 & $(0.61)$ & 0.084 & -0.004 & $(0.019)$ & 0.673 & -0.019 & $(0.027)$ & 0.471 \\
\hline Zinc $(\Delta \mathrm{mg} / \mathrm{d})$ & 0.26 & $(0.65)$ & 0.684 & -0.20 & $(1.00)$ & 0.844 & 0.082 & $(0.044)$ & 0.062 & 0.082 & $(0.044)$ & 0.062 \\
\hline Copper $(\Delta \mathrm{mg} / \mathrm{d})$ & -7.34 & $(4.45)$ & 0.100 & -10.4 & $(5.39)$ & 0.055 & -0.089 & $(0.197)$ & 0.650 & -0.133 & $(0.239)$ & 0.578 \\
\hline Manganese $(\Delta \mathrm{mg} / \mathrm{d})$ & -2.43 & $(1.02)$ & 0.018 & -2.19 & $(1.07)$ & $0.042 *$ & 0.000 & $(0.001)$ & 0.565 & -0.001 & $(0.001)$ & 0.272 \\
\hline Vitamin A (retinol equivalent) $(\Delta \mu \mathrm{g} / \mathrm{d})$ & 0.00 & $(0.00)$ & 0.582 & 0.00 & $(0.00)$ & 0.436 & 0.000 & $(0.000)$ & 0.980 & 0.000 & $(0.000)$ & 0.938 \\
\hline $\operatorname{Retinol}(\Delta \mu \mathrm{g} / \mathrm{d})$ & 0.00 & $(0.00)$ & 0.618 & 0.00 & $(0.00)$ & 0.501 & 0.000 & $(0.000)$ & 0.940 & 0.000 & $(0.000)$ & 0.898 \\
\hline$\beta$-carotene equivalente $(\Delta \mu \mathrm{g} / \mathrm{d})$ & 0.00 & $(0.00)$ & 0.885 & 0.00 & $(0.00)$ & 0.726 & 0.000 & $(0.000)$ & 0.992 & 0.000 & $(0.000)$ & 0.944 \\
\hline $\operatorname{Vitamin} \mathrm{D}(\Delta \mu \mathrm{g} / \mathrm{d})$ & 0.07 & $(0.08)$ & 0.423 & 0.09 & $(0.12)$ & 0.452 & -0.003 & $(0.004)$ & 0.375 & -0.010 & $(0.005)$ & 0.063 \\
\hline$\beta$-tocopherol $(\Delta \mathrm{mg} / \mathrm{d})$ & 0.44 & $(0.43)$ & 0.307 & 0.34 & $(0.62)$ & 0.578 & -0.023 & $(0.019)$ & 0.219 & -0.061 & $(0.027)$ & 0.023 * \\
\hline Vitamin $\mathrm{K}(\Delta \mu \mathrm{g} / \mathrm{d})$ & -0.01 & $(0.01)$ & 0.131 & -0.01 & $(5.85)$ & 0.050 * & 0.000 & $(0.000)$ & 0.360 & 0.000 & $(0.000)$ & 0.395 \\
\hline Thiamin $(\Delta \mathrm{mg} / \mathrm{d})$ & -2.58 & $(5.27)$ & 0.625 & -11.9 & $(7.48)$ & 0.111 & 0.112 & $(0.232)$ & 0.629 & 0.085 & $(0.330)$ & 0.797 \\
\hline Riboflavin $(\Delta \mathrm{mg} / \mathrm{d})$ & 0.12 & $(0.12)$ & 0.339 & 0.19 & $(0.18)$ & 0.294 & 0.134 & $(0.112)$ & 0.230 & 0.184 & $(0.151)$ & 0.224 \\
\hline $\operatorname{Niacin}(\Delta \mathrm{mg} / \mathrm{d})$ & 0.12 & $(0.15)$ & 0.417 & 0.18 & $(0.27)$ & 0.522 & -0.001 & $(0.007)$ & 0.930 & -0.010 & $(0.012)$ & 0.426 \\
\hline Vitamin $\mathrm{B}_{6}(\Delta \mathrm{mg} / \mathrm{d})$ & 0.54 & $(2.61)$ & 0.837 & -2.14 & $(4.48)$ & 0.632 & -0.117 & $(0.114)$ & 0.307 & -0.474 & (0.195) & 0.015 * \\
\hline Vitamin $\mathrm{B}_{12}(\Delta \mathrm{mg} / \mathrm{d})$ & 0.12 & $(0.12)$ & 0.339 & 0.19 & $(0.18)$ & 0.294 & -0.006 & $(0.005)$ & 0.260 & -0.020 & $(0.008)$ & 0.015 * \\
\hline Folate $(\Delta \mu \mathrm{g} / \mathrm{d})$ & -0.01 & $(0.01)$ & 0.116 & -0.02 & $(0.01)$ & 0.045 * & 0.000 & $(0.000)$ & 0.783 & 0.000 & $(0.000)$ & 0.655 \\
\hline Pantothenic acid $(\Delta \mathrm{mg} / \mathrm{d})$ & -1.16 & $(0.70)$ & 0.099 & -3.43 & $(1.02)$ & $0.001^{* *}$ & 0.049 & $(0.031)$ & 0.115 & 0.089 & $(0.046)$ & 0.051 \\
\hline Vitamin $C(\Delta \mathrm{mg} / \mathrm{d})$ & -0.02 & $(0.02)$ & 0.448 & -0.02 & $(0.02)$ & 0.341 & -0.002 & $(0.001)$ & 0.068 & -0.002 & $(0.001)$ & 0.057 \\
\hline Saturated fat $(\Delta \mathrm{g} / \mathrm{d})$ & -0.06 & $(0.21)$ & 0.781 & -0.48 & $(0.35)$ & 0.170 & 0.013 & $(0.009)$ & 0.165 & 0.025 & $(0.015)$ & 0.105 \\
\hline Monounsaturated fat $(\Delta \mathrm{g} / \mathrm{d})$ & 0.16 & $(0.17)$ & 0.325 & 1.34 & $(0.68)$ & 0.050 * & 0.006 & $(0.007)$ & 0.431 & 0.016 & $(0.030)$ & 0.605 \\
\hline Polyunsaturated fat $(\Delta \mathrm{g} / \mathrm{d})$ & 0.16 & $(0.24)$ & 0.497 & 0.03 & $(0.42)$ & 0.938 & -0.001 & $(0.011)$ & 0.900 & -0.019 & $(0.018)$ & 0.287 \\
\hline $\mathrm{n}-3$ polyunsaturated fat $(\Delta \mathrm{g} / \mathrm{d})$ & 0.71 & $(0.82)$ & 0.389 & 0.57 & $(1.22)$ & 0.640 & -0.034 & $(0.036)$ & 0.347 & -0.115 & $(0.053)$ & 0.031 \\
\hline n- 6 polyunsaturated fat $(\Delta \mathrm{g} / \mathrm{d})$ & 0.14 & $(0.30)$ & 0.642 & -0.10 & $(0.48)$ & 0.836 & 0.004 & $(0.013)$ & 0.770 & -0.004 & $(0.021)$ & 0.864 \\
\hline Cholesterol $(\Delta \mathrm{mg} / \mathrm{d})$ & 0.00 & $(0.01)$ & 0.772 & 0.00 & $(0.01)$ & 0.850 & 0.000 & $(0.000)$ & 0.318 & 0.000 & $(0.000)$ & 0.438 \\
\hline
\end{tabular}


Table 4. Cont.

\begin{tabular}{|c|c|c|c|c|c|c|c|c|c|c|c|c|}
\hline \multirow{4}{*}{$\begin{array}{c}\text { Characteristics } \\
\text { Micronutrient: } \\
\text { Total dietary fiber }(\Delta \mathrm{g} / \mathrm{d})\end{array}$} & \multicolumn{6}{|c|}{ Change in VFA } & \multicolumn{6}{|c|}{ Change in BMI } \\
\hline & \multicolumn{3}{|c|}{ Adjusted by Model 1} & \multicolumn{3}{|c|}{ Adjusted by Model 2} & \multicolumn{3}{|c|}{ Adjusted by Model 3} & \multicolumn{3}{|c|}{ Adjusted by Model 4} \\
\hline & \multicolumn{2}{|c|}{$\beta$ (s.e.) } & \multirow{2}{*}{$\begin{array}{c}p \\
0.022\end{array}$} & \multicolumn{2}{|c|}{$\beta$ (s.e.) } & \multirow{2}{*}{$\frac{p}{0.013^{*}}$} & \multicolumn{2}{|c|}{$\beta$ (s.e.) } & \multirow{2}{*}{$\begin{array}{c}p \\
0.320\end{array}$} & \multicolumn{2}{|c|}{$\beta$ (s.e.) } & \multirow{2}{*}{$\begin{array}{c}p \\
0.298\end{array}$} \\
\hline & -0.64 & $(0.28)$ & & -0.73 & $(0.29)$ & & -0.012 & $(0.012)$ & & -0.014 & $(0.013)$ & \\
\hline Soluble dietary fiber $(\Delta \mathrm{g} / \mathrm{d})$ & -2.31 & $(0.94)$ & 0.014 & -2.59 & $(0.97)$ & $0.007^{* *}$ & -0.046 & $(0.041)$ & 0.264 & -0.050 & $(0.043)$ & 0.245 \\
\hline Insoluble dietary fiber $(\Delta \mathrm{g} / \mathrm{d})$ & -0.74 & $(0.41)$ & 0.070 & -0.82 & $(0.43)$ & 0.055 & -0.017 & $(0.018)$ & 0.333 & -0.019 & $(0.019)$ & 0.313 \\
\hline $\operatorname{Alcohol}(\Delta \mathrm{g} / \mathrm{d})$ & -0.03 & $(0.05)$ & 0.584 & -0.01 & $(0.05)$ & 0.908 & 0.001 & $(0.002)$ & 0.541 & 0.001 & $(0.002)$ & 0.497 \\
\hline Daidzein $(\Delta \mathrm{mg} / \mathrm{d})$ & -0.11 & $(0.09)$ & 0.205 & -0.14 & $(0.09)$ & 0.126 & 0.003 & $(0.004)$ & 0.405 & 0.003 & $(0.004)$ & 0.428 \\
\hline Genistein $(\Delta \mathrm{mg} / \mathrm{d})$ & -0.07 & $(0.05)$ & 0.213 & -0.08 & $(0.05)$ & 0.132 & 0.002 & $(0.002)$ & 0.414 & 0.002 & $(0.002)$ & 0.438 \\
\hline
\end{tabular}

$p<0.05$ and $<0.01$ are indicated by "** and "***" respectively. $\beta$ was regression coefficient. a Model 1 was adjusted by age, gender, VFA, and intake amount of each macronutrient (protein, fat, and carbohydrate) at baseline, and change in energy intake, amount of exercise, BMI, WC, and smoking habit. ${ }^{b}$ Model 2 was adjusted by age, sex, VFA, and intake amount of each macronutrient (protein, fat, and carbohydrate) at baseline, and change in intake of protein, fat, and carbohydrate; amount of exercise; BMI; WC; smoking habit; and amount of energy. ${ }^{\mathrm{c}}$ Model 3 was adjusted by age, gender, BMI, and intake amount of each macronutrient (protein, fat, and carbohydrate) at baseline, and change in energy intake, amount of exercise, VFA, WC, and smoking habit. ${ }^{\mathrm{d}}$ Model 4 was adjusted by age, gender, BMI, and amount of each macronutrient at baseline, and change in intake of protein, fat, and carbohydrate; amount of exercise; VFA; WC; smoking habit; and amount of energy. 


\section{Discussion}

We investigated the association of eating habits with change in VFA over 2 years. Baseline surveys indicated that VFA was significantly associated with other metabolic syndrome factors. These findings supported those of previous reports that VFA is a predictor of cardiovascular disease [4-7]. Regarding dietary habits, some nutrients were significantly associated with VFA in only men or in only women, but no nutrients were associated with VFA, regardless of sex, in our baseline analysis.

In our longitudinal analyses, we found no significant relationship between change in VFA and energy intake or amount of each macronutrient (protein, fat, and carbohydrate) ingested, even after 2 years, whether or not adjustments were made for changes in energy intake. Generally, habitual diets in humans are a mixture of "macronutrients" and "micronutrients"; therefore, we hypothesized that these two elements are mutual "confounding factors", and thus determined their associations. The macronutrients were significantly highly correlated with all of the micronutrients except for alcohol (Table 3). Therefore, we adjusted for macronutrients when assessing the associations between micronutrients and VFA. In Model 1, which included changes in energy intake but not macronutrient intake, only two micronutrients: soluble dietary fiber and manganese, were significantly associated with VFA. In Model 2, which included changes in macronutrient intake but not energy intake, nine macronutrients were significantly associated with VFA (Table 4). Similar results were found when using BMI as an index of obesity. These findings suggest that different results may be obtained when investigating the association of micronutrients with VFA depending on whether adjustments are made for macronutrients. Although further studies are needed, this might be one reason that previous studies reported only a few macronutrients associated with VFA [21-23].

To gain insight into the importance of adjusting for macronutrients when investigating the association between micronutrients and VFA, the validity of our observation of each nutrient is discussed here and compared with the findings of previous reports. With respect to water-soluble dietary fiber, inconsistent conclusions were reported among previous studies [21,23,39,40]. A study of 85 individuals [23] reported no correlation; however, in a survey of 1114 individuals [21], those individuals who increased their intake of soluble dietary fiber had reduced visceral fat. In addition, an intervention trial reported that dietary fiber [39] and $\beta$-glucan [40], which is a type of soluble dietary fiber, are effective for reducing VFA, supporting our results. With regard to folic acid, no significant relationship was identified in an epidemiologic study [41], but an intervention study reported that folic acid intake was effective for reducing visceral fat [42]. The association between folic acid and VFA observed in the present study differed from the previous epidemiology study [41], which might be due to the differences in our analytical method. Magnesium reportedly contributes to insulin resistance and the risk of metabolic syndrome in association with visceral fat [43], possibly supporting our observations. With regard to manganese, intake of manganese was inversely correlated with abdominal obesity in a cross-sectional study [44]. Although an interventional test is required, our result is consistent with this report. With regard to vitamin $\mathrm{K}$, which had a borderline $p$-value $(p=0.05)$, an interventional study reported that it is related to improvement of WC, and highly negatively correlated with VFA [45], supporting our results. No previous studies of pantothenic acid in relation to VFA have been reported. An intervention study of this association would be useful. In cross-sectional studies, no significant association between monounsaturated fatty acids, which had a borderline $p$-value $(p=0.05)$, and VFA are reported [21,46]. A longitudinal study found a significant inverse association [47], which supports the present results. Calcium and alcohol are reported to be significantly associated with VFA, but our study did not confirm this finding. Both a cross-sectional study [41] and a long-term study [22] reported that high or increased visceral fat is associated with a low calcium intake. While some interventional studies have evaluated the association between calcium and vitamin D3 intake with visceral fat $[48,49]$, there are no human intervention studies investigating the association between calcium alone and visceral fat. Some cross-sectional studies report a significant relationship of alcohol with VFA [10,12,50,51], whereas others report no significant relationship [41,46]. An intervention study [52] found no significant relationship. Thus, the inconsistencies in the associations between 
micronutrients and VFA might be clarified by adjusting for macronutrients as confounding factors, even in epidemiologic studies.

On the other hand, the opposite associations are well known in animal meat. These associations are consistently reported for foodstuffs [53-56]. In our observations, however, the micronutrients associated with VFA and with BMI were completely different. This is the first study, to our knowledge, to evaluate nutrients associated with both VFA and with BMI. Thus, there might be unique associations of micronutrients with VFA in contrast to a simple obesity index like BMI. Of note, visceral fat is reported to be a higher risk factor for all-cause mortality, including cardiovascular disease, than BMI [4-7].

A limitation of the present study is that it was a 2-year longitudinal study, and the causality between food and obesity is unclear. As this study was performed in a limited country, region, and race, the reproducibility should be confirmed in a different country and/or race. VFA was measured using an impedance method rather than the gold standard method of $\mathrm{CT}$, but the results of the impedance method correlate highly with those of CT [36]. The BDHQ estimates the nutritional components for only a small number of nutrients. Furthermore, a questionnaire regarding nutritional compounds during the preceding months is flawed with uncertainties.

The present study is a longitudinal study and reports on macronutrients associated with visceral fat using a comprehensive dataset of dietary micronutrients obtained with the BDHQ. Moreover, to further clarify the association of micronutrients with VFA, macronutrients were considered as confounders. Therefore, eight micronutrients were found to be significantly associated with VFA.

As visceral fat is a predictive factor of cardiovascular disease, it is important to analyze the factors involved in the development of visceral fat in healthy individuals. Thus, our observation in over 600 relatively healthy individuals is expected to be useful for proposing a healthy diet.

\section{Conclusions}

Seven micronutrients abundant nutrients in vegetable diets were found to potentially contribute to suppressive effects against the accumulation of visceral fat. Only one micronutrient, monounsaturated fat, was significantly positively associated with the accumulation of visceral fat. Analyses that adjust for macronutrients as confounders might be an alternative for evaluating the association of micronutrients with VFA.

Author Contributions: N.O. analyzed and interpreted the data. N.O. and S.S. designed the analyses and contributed to the manuscript. T.Y., M.K., I.T., K.S., and S.N. contributed to the data acquisition. S.S., M.K., T.Y., Y.K., S.I., K.I., and S.N. contributed to the data interpretation. S.N. is the guarantor of this work and takes responsibility for the integrity and accuracy of the data. All authors reviewed and edited the manuscript and approved the final version of the manuscript.

Funding: This work was supported by JST, Center of Innovation Program (JPMJCE1302), and Kao Co. (Tokyo, Japan).

Acknowledgments: We thank Haruo Nakamura from National Defense Medical College who was the physician.

Conflicts of Interest: The authors declare no conflict of interest. N.O., S.S., T.Y., M.K., and Y.K. are employees of the Kao Corporation.

\section{References}

1. World Health Organization. Obesity: preventing and managing the global epidemic. In World Health Organization Technical Report Series 894; World Health Organization: Geneva, Switzerland, 2000; Volume 1-12, pp. 1-253.

2. Beunza, J.J.; Toledo, E.; Hu, F.B.; Bes-Rastrollo, M.; Serrano-Martinez, M.; Sanchez-Villegas, A.; Martinez, J.A.; Martinez-Gonzalez, M.A. Adherence to the Mediterranean diet, long-term weight change, and incident overweight or obesity: The Seguimiento Universidad de Navarra (SUN) cohort. Am. J. Clin. Nutr. 2010, 92, 1484-1493. [CrossRef] [PubMed]

3. GBD 2017 Diet Collaborators. Health effects of dietary risks in 195 countries, 1990-2017: A systematic analysis for the Global Burden of Disease Study 2017. Lancet 2019, 393, 1958-1972. [CrossRef] 
4. Tchernof, A.; Despres, J.P. Pathophysiology of human visceral obesity: An update. Physiol. Rev. 2013, 93, 359-404. [CrossRef] [PubMed]

5. Kuk, J.L.; Katzmarzyk, P.T.; Nichaman, M.Z.; Church, T.S.; Blair, S.N.; Ross, R. Visceral fat is an independent predictor of all-cause mortality in men. Obesity 2006, 14, 336-341. [CrossRef]

6. McNeely, M.J.; Shofer, J.B.; Leonetti, D.L.; Fujimoto, W.Y.; Boyko, E.J. Associations among visceral fat, all-cause mortality, and obesity-related mortality in Japanese Americans. Diabetes Care 2012, 35, $296-298$.

7. Koster, A.; Murphy, R.A.; Eiriksdottir, G.; Aspelund, T.; Sigurdsson, S.; Lang, T.F.; Gudnason, V.; Launer, L.J.; Harris, T.B. Fat distribution and mortality: The AGES-Reykjavik Study. Obesity 2015, 23, 893-897. [CrossRef]

8. Matsushita, Y.; Nakagawa, T.; Yamamoto, S.; Takahashi, Y.; Yokoyama, T.; Noda, M.; Mizoue, T. Associations of visceral and subcutaneous fat areas with the prevalence of metabolic risk factor clustering in 6,292 Japanese individuals: The Hitachi Health Study. Diabetes Care 2010, 33, 2117-2119. [CrossRef]

9. Shah, R.V.; Murthy, V.L.; Abbasi, S.A.; Blankstein, R.; Kwong, R.Y.; Goldfine, A.B.; Jerosch-Herold, M.; Lima, J.A.; Ding, J.; Allison, M.A. Visceral adiposity and the risk of metabolic syndrome across body mass index: The MESA Study. JACC Cardiovasc. Imaging 2014, 7, 1221-1235. [CrossRef]

10. Kim, K.H.; Oh, S.W.; Kwon, H.; Park, J.H.; Choi, H.; Cho, B. Alcohol consumption and its relation to visceral and subcutaneous adipose tissues in healthy male Koreans. Ann. Nutr. Metab. 2012, 60, 52-61. [CrossRef]

11. Mure, K.; Maeda, S.; Mukoubayashi, C.; Mugitani, K.; Iwane, M.; Kinoshita, F.; Mohara, O.; Takeshita, T. Habitual coffee consumption inversely associated with metabolic syndrome-related biomarkers involving adiponectin. Nutrition 2013, 29, 982-987.

12. Kondoh, T.; Takase, H.; Yamaguchi, T.F.; Ochiai, R.; Katashima, M.; Katsuragi, Y.; Sakane, N. Association of dietary factors with abdominal subcutaneous and visceral adiposity in Japanese men. Obes. Res. Clin. Pract. 2014, 8, e16-e25. [CrossRef] [PubMed]

13. Ruttgers, D.; Fischer, K.; Koch, M.; Lieb, W.; Muller, H.P.; Jacobs, G.; Kassubek, J.; Nothlings, U. Association of food consumption with total volumes of visceral and subcutaneous abdominal adipose tissue in a Northern German population. Br. J. Nutr. 2015, 114, 1929-1940. [CrossRef] [PubMed]

14. Doubelt, I.; Totosy de Zepetnek, J.; MacDonald, M.J.; Atkinson, S.A. Influences of nutrition and adiposity on bone mineral density in individuals with chronic spinal cord injury: A cross-sectional, observational study. Bone Rep. 2015, 2, 26-31. [CrossRef] [PubMed]

15. Sluijs, I.; Beulens, J.W.; Grobbee, D.E.; van der Schouw, Y.T. Dietary carotenoid intake is associated with lower prevalence of metabolic syndrome in middle-aged and elderly men. J. Nutr. 2009, 139, 987-992. [CrossRef] [PubMed]

16. Loos, R.J.; Rankinen, T.; Leon, A.S.; Skinner, J.S.; Wilmore, J.H.; Rao, D.C.; Bouchard, C. Calcium intake is associated with adiposity in Black and White men and White women of the HERITAGE Family Study. J. Nutr. 2004, 134, 1772-1778. [CrossRef]

17. Parikh, S.; Pollock, N.K.; Bhagatwala, J.; Guo, D.H.; Gutin, B.; Zhu, H.; Dong, Y. Adolescent fiber consumption is associated with visceral fat and inflammatory markers. J. Clin. Endocrinol. Metab. 2012, 97, E1451-E1457. [CrossRef]

18. Pollock, N.K.; Bundy, V.; Kanto, W.; Davis, C.L.; Bernard, P.J.; Zhu, H.; Gutin, B.; Dong, Y. Greater fructose consumption is associated with cardiometabolic risk markers and visceral adiposity in adolescents. J. Nutr. 2012, 142, 251-257. [CrossRef]

19. Takase, H.; Sakane, N.; Morimoto, T.; Uchida, T.; Mori, K.; Katashima, M.; Katsuragi, Y. Development of a Dietary Factor Assessment Tool for Evaluating Associations between Visceral Fat Accumulation and Major Nutrients in Japanese Adults. J. Obes. 2019, 2019, 9497861. [CrossRef]

20. Bautista-Castano, I.; Sanchez-Villegas, A.; Estruch, R.; Martinez-Gonzalez, M.A.; Corella, D.; Salas-Salvado, J.; Covas, M.I.; Schroder, H.; Alvarez-Perez, J.; Quilez, J.; et al. Changes in bread consumption and 4-year changes in adiposity in Spanish subjects at high cardiovascular risk. Br. J. Nutr. 2013, 110, 337-346. [CrossRef]

21. Hairston, K.G.; Vitolins, M.Z.; Norris, J.M.; Anderson, A.M.; Hanley, A.J.; Wagenknecht, L.E. Lifestyle factors and 5-year abdominal fat accumulation in a minority cohort: The IRAS Family Study. Obesity 2012, 20, 421-427. [CrossRef]

22. Bush, N.C.; Alvarez, J.A.; Choquette, S.S.; Hunter, G.R.; Oster, R.A.; Darnell, B.E.; Gower, B.A. Dietary calcium intake is associated with less gain in intra-abdominal adipose tissue over 1 year. Obesity 2010, 18, 2101-2104. [CrossRef] [PubMed] 
23. Davis, J.N.; Alexander, K.E.; Ventura, E.E.; Toledo-Corral, C.M.; Goran, M.I. Inverse relation between dietary fiber intake and visceral adiposity in overweight Latino youth. Am. J. Clin. Nutr. 2009, 90, 1160-1166. [CrossRef] [PubMed]

24. Koga, R.; Tanaka, M.; Tsuda, H.; Imai, K.; Abe, S.; Masuda, T.; Iwamoto, M.; Nakazono, E.; Kamohara, T.; Sakata, T. Daily exercise fluctuations and dietary patterns during training predict visceral fat regain in obese women. Am. J. Med. Sci. 2008, 336, 450-457. [CrossRef] [PubMed]

25. Tanaka, S.; Horimai, C.; Katsukawa, F. Ethnic differences in abdominal visceral fat accumulation between Japanese, African-Americans, and Caucasians: A meta-analysis. Acta Diabetol. 2003, 40 (Suppl. 1), S302-S304. [CrossRef]

26. Daimon, M.; Kamba, A.; Murakami, H.; Mizushiri, S.; Osonoi, S.; Matsuki, K.; Sato, E.; Tanabe, J.; Takayasu, S.; Matsuhashi, Y.; et al. Dominance of the hypothalamus-pituitary-adrenal axis over the renin-angiotensin-aldosterone system is a risk factor for decreased insulin secretion. Sci. Rep. 2017, 7, 11360. [CrossRef]

27. Iino, C.; Shimoyama, T.; Chinda, D.; Arai, T.; Chiba, D.; Nakaji, S.; Fukuda, S. Infection of Helicobacter pylori and Atrophic Gastritis Influence Lactobacillus in Gut Microbiota in a Japanese Population. Front. Immunol. 2018, 9, 712. [CrossRef]

28. Iino, C.; Shimoyama, T.; Iino, K.; Yokoyama, Y.; Chinda, D.; Sakuraba, H.; Fukuda, S.; Nakaji, S. Daidzein Intake Is Associated with Equol Producing Status through an Increase in the Intestinal Bacteria Responsible for Equol Production. Nutrients 2019, 11, 433. [CrossRef]

29. Kumagai, G.; Wada, K.; Kudo, H.; Asari, T.; Chiba, D.; Ota, S.; Takeda, O.; Koyama, K.; Nakaji, S.; Ishibashi, Y. Associations between cervical disc degeneration and muscle strength in a cross-sectional population-based study. PLoS ONE 2019, 14, e0210802. [CrossRef]

30. Sasaki, S.; Yanagibori, R.; Amano, K. Self-Administered diet history questionnaire developed for health education: A relative validation of the test-version by comparison with 3-day diet record in women. J. Epidemiol. 1998, 8, 203-215. [CrossRef]

31. Sasaki, S.; Ushio, F.; Amano, K.; Morihara, M.; Todoriki, O.; Uehara, Y.; Toyooka, E. Serum biomarker-based validation of a self-administered diet history questionnaire for Japanese subjects. J. Nutr. Sci. Vitaminol. 2000, 46, 285-296. [CrossRef]

32. Kobayashi, S.; Honda, S.; Murakami, K.; Sasaki, S.; Okubo, H.; Hirota, N.; Notsu, A.; Fukui, M.; Date, C. Both comprehensive and brief self-administered diet history questionnaires satisfactorily rank nutrient intakes in Japanese adults. J. Epidemiol. 2012, 22, 151-159. [CrossRef] [PubMed]

33. Kobayashi, S.; Murakami, K.; Sasaki, S.; Okubo, H.; Hirota, N.; Notsu, A.; Fukui, M.; Date, C. Comparison of relative validity of food group intakes estimated by comprehensive and brief-type self-administered diet history questionnaires against $16 \mathrm{~d}$ dietary records in Japanese adults. Public Health Nutr. 2011, 14, 1200-1211. [CrossRef] [PubMed]

34. Sukenobe, Y.; Terauchi, M.; Hirose, A.; Hirano, M.; Akiyoshi, M.; Kato, K.; Miyasaka, N. Normal/high-fat milk consumption is associated with higher lean body and muscle mass in Japanese women aged between 40 and 60 years: A cross-sectional study. BMC Womens Health 2018, 18, 32. [CrossRef] [PubMed]

35. Tsuruya, K.; Fukuma, S.; Wakita, T.; Ninomiya, T.; Nagata, M.; Yoshida, H.; Fujimi, S.; Kiyohara, Y.; Kitazono, T.; Uchida, K.; et al. Dietary patterns and clinical outcomes in hemodialysis patients in Japan: A cohort study. PLoS ONE 2015, 10, e0116677. [CrossRef]

36. Ryo, M.; Maeda, K.; Onda, T.; Katashima, M.; Okumiya, A.; Nishida, M.; Yamaguchi, T.; Funahashi, T.; Matsuzawa, Y.; Nakamura, T.; et al. A new simple method for the measurement of visceral fat accumulation by bioelectrical impedance. Diabetes Care 2005, 28, 451-453. [CrossRef]

37. Ainsworth, B.E.; Haskell, W.L.; Herrmann, S.D.; Meckes, N.; Bassett, D.R., Jr.; Tudor-Locke, C.; Greer, J.L.; Vezina, J.; Whitt-Glover, M.C.; Leon, A.S. 2011 Compendium of Physical Activities: A second update of codes and MET values. Med. Sci. Sports Exerc. 2011, 43, 1575-1581. [CrossRef]

38. Examination Committee of Criteria for 'Obesity Disease' in Japan. Japan Society for the Study of Obesity: New criteria for 'obesity disease' in Japan. Circ. J. 2002, 66, 987-992. [CrossRef]

39. Ventura, E.; Davis, J.; Byrd-Williams, C.; Alexander, K.; McClain, A.; Lane, C.J.; Spruijt-Metz, D.; Weigensberg, M.; Goran, M. Reduction in risk factors for type 2 diabetes mellitus in response to a low-sugar, high-fiber dietary intervention in overweight Latino adolescents. Arch. Pediatr. Adolesc. Med. 2009, 163, 320-327. [CrossRef] 
40. Aoe, S.; Ichinose, Y.; Kohyama, N.; Komae, K.; Takahashi, A.; Abe, D.; Yoshioka, T.; Yanagisawa, T. Effects of high beta-glucan barley on visceral fat obesity in Japanese individuals: A randomized, double-blind study. Nutrition 2017, 42, 1-6. [CrossRef]

41. Bailey, B.W.; Sullivan, D.K.; Kirk, E.P.; Donnelly, J.E. Dietary predictors of visceral adiposity in overweight young adults. Br. J. Nutr. 2010, 103, 1702-1705. [CrossRef]

42. Buettner, R.; Bettermann, I.; Hechtl, C.; Gabele, E.; Hellerbrand, C.; Scholmerich, J.; Bollheimer, L.C. Dietary folic acid activates AMPK and improves insulin resistance and hepatic inflammation in dietary rodent models of the metabolic syndrome. Horm. Metab. Res. 2010, 42, 769-774. [CrossRef] [PubMed]

43. Rayssiguier, Y.; Gueux, E.; Nowacki, W.; Rock, E.; Mazur, A. High fructose consumption combined with low dietary magnesium intake may increase the incidence of the metabolic syndrome by inducing inflammation. Magnes. Res. 2006, 19, 237-243. [PubMed]

44. Zhou, B.; Su, X.; Su, D.; Zeng, F.; Wang, M.H.; Huang, L.; Huang, E.; Zhu, Y.; Zhao, D.; He, D.; et al. Dietary intake of manganese and the risk of the metabolic syndrome in a Chinese population. Br. J. Nutr. 2016, 116, 853-863. [CrossRef] [PubMed]

45. Knapen, M.H.J.; Jardon, K.M.; Vermeer, C. Vitamin K-induced effects on body fat and weight: Results from a 3-year vitamin K2 intervention study. Eur. J. Clin. Nutr. 2018, 72, 136-141. [CrossRef] [PubMed]

46. Larson, D.E.; Hunter, G.R.; Williams, M.J.; Kekes-Szabo, T.; Nyikos, I.; Goran, M.I. Dietary fat in relation to body fat and intraabdominal adipose tissue: A cross-sectional analysis. Am. J. Clin. Nutr. 1996, 64, 677-684. [CrossRef] [PubMed]

47. Moslehi, N.; Ehsani, B.; Mirmiran, P.; Hojjat, P.; Azizi, F. Association of Dietary Proportions of Macronutrients with Visceral Adiposity Index: Non-Substitution and Iso-Energetic Substitution Models in a Prospective Study. Nutrients 2015, 7, 8859-8870. [CrossRef] [PubMed]

48. Zhu, W.; Cai, D.; Wang, Y.; Lin, N.; Hu, Q.; Qi, Y.; Ma, S.; Amarasekara, S. Calcium plus vitamin D3 supplementation facilitated fat loss in overweight and obese college students with very-low calcium consumption: A randomized controlled trial. Nutr. J. 2013, 12, 8. [CrossRef]

49. Rosenblum, J.L.; Castro, V.M.; Moore, C.E.; Kaplan, L.M. Calcium and vitamin D supplementation is associated with decreased abdominal visceral adipose tissue in overweight and obese adults. Am. J. Clin. Nutr. 2012, 95, 101-108. [CrossRef]

50. Molenaar, E.A.; Massaro, J.M.; Jacques, P.F.; Pou, K.M.; Ellison, R.C.; Hoffmann, U.; Pencina, K.; Shadwick, S.D.; Vasan, R.S.; O'Donnell, C.J.; et al. Association of lifestyle factors with abdominal subcutaneous and visceral adiposity: The Framingham Heart Study. Diabetes Care 2009, 32, 505-510. [CrossRef]

51. Sumi, M.; Hisamatsu, T.; Fujiyoshi, A.; Kadota, A.; Miyagawa, N.; Kondo, K.; Kadowaki, S.; Suzuki, S.; Torii, S.; Zaid, M.; et al. Association of alcohol consumption with fat deposition in a community-based sample of Japanese men: The Shiga Epidemiological Study of Subclinical Atherosclerosis (SESSA). J. Epidemiol. 2018, 29. [CrossRef]

52. Beulens, J.W.; van Beers, R.M.; Stolk, R.P.; Schaafsma, G.; Hendriks, H.F. The effect of moderate alcohol consumption on fat distribution and adipocytokines. Obesity 2006, 14, 60-66. [CrossRef] [PubMed]

53. Zhan, J.; Liu, Y.J.; Cai, L.B.; Xu, F.R.; Xie, T.; He, Q.Q. Fruit and vegetable consumption and risk of cardiovascular disease: A meta-analysis of prospective cohort studies. Crit. Rev. Food Sci. Nutr. 2017, 57, 1650-1663. [CrossRef] [PubMed]

54. Lampe, J.W. Health effects of vegetables and fruit: Assessing mechanisms of action in human experimental studies. Am. J. Clin. Nutr. 1999, 70 (Suppl. 3), 475S-490S. [CrossRef] [PubMed]

55. Kant, A.K.; Schatzkin, A.; Graubard, B.I.; Schairer, C. A prospective study of diet quality and mortality in women. JAMA 2000, 283, 2109-2115. [CrossRef] [PubMed]

56. Osler, M.; Heitmann, B.L.; Gerdes, L.U.; Jorgensen, L.M.; Schroll, M. Dietary patterns and mortality in Danish men and women: A prospective observational study. Br. J. Nutr. 2001, 85, 219-225. [CrossRef] [PubMed]

(C) 2019 by the authors. Licensee MDPI, Basel, Switzerland. This article is an open access article distributed under the terms and conditions of the Creative Commons Attribution (CC BY) license (http://creativecommons.org/licenses/by/4.0/). 